\title{
A Sufficient Condition for Voting Rules to Be Frequently Manipulable
}

\author{
Lirong Xia \\ Department of Computer Science Duke \\ University \\ Durham, NC 27708, USA \\ Ixia@cs.duke.edu
}

\author{
Vincent Conitzer \\ Department of Computer Science Duke \\ University \\ Durham, NC 27708, USA \\ conitzer@cs.duke.edu
}

\begin{abstract}
The Gibbard-Satterthwaite Theorem states that (in unrestricted settings) any reasonable voting rule is manipulable. Recently, a quantitative version of this theorem was proved by Ehud Friedgut, Gil Kalai, and Noam Nisan: when the number of alternatives is three, for any neutral voting rule that is far from any dictatorship, there exists a voter such that a random manipulation-that is, the true preferences and the strategic vote are all drawn i.i.d., uniformly at random-will succeed with a probability of $\Omega\left(\frac{1}{n}\right)$, where $n$ is the number of voters. However, it seems that the techniques used to prove this theorem can not be fully extended to more than three alternatives. In this paper, we give a more limited result that does apply to four or more alternatives. We give a sufficient condition for a voting rule to be randomly manipulable with a probability of $\Omega\left(\frac{1}{n}\right)$ for at least one voter, when the number of alternatives is held fixed. Specifically, our theorem states that if a voting rule $r$ satisfies 1. homogeneity, 2. anonymity, 3 . non-imposition, 4. a cancelingout condition, and 5. there exists a stable profile that is still stable after one given alternative is uniformly moved to different positions; then there exists a voter such that a random manipulation for that voter will succeed with a probability of $\Omega\left(\frac{1}{n}\right)$. We show that many common voting rules satisfy these conditions, for example any positional scoring rule, Copeland, STV, maximin, and ranked pairs.
\end{abstract}

\section{Categories and Subject Descriptors}

J.4 [Computer Applications]: Social and Behavioral SciencesEconomics; I.2.11 [Distributed Artificial Intelligence]: Multiagent Systems

\section{General Terms}

Algorithms, Economics, Theory

\section{Keywords}

Computational social choice, complexity of manipulation, mechanism design

Permission to make digital or hard copies of all or part of this work for personal or classroom use is granted without fee provided that copies are not made or distributed for profit or commercial advantage and that copies bear this notice and the full citation on the first page. To copy otherwise, to republish, to post on servers or to redistribute to lists, requires prior specific permission and/or a fee.

EC'08, July 8-12, 2008, Chicago, Illinois, USA.

Copyright 2008 ACM 978-1-60558-169-9/08/07 ...\$5.00.

\section{INTRODUCTION}

If a group of agents needs to decide among a set of alternatives, they can do so by voting over the alternatives. First, agents are asked to submit their preferences (usually in the form of linear orders over the alternatives); then, the winner is selected based on the reported preferences according to a voting rule. One complication in this process is manipulation, that is, a voter can sometimes obtain a better result for herself by declaring her preferences insincerely. One may try to prevent this by creating a rule that is strategy-proof, that is, a rule in which reporting one's true preferences is always optimal. Unfortunately, when there are three or more alternatives and preferences are unrestricted, no rule that satisfies non-imposition (for every alternative, there exist votes that would make that alternative win) and non-dictatorship (the rule does not simply always choose the most-preferred alternative of a single fixed voter) is strategy-proof, that is, a manipulation always exists. This fundamental impossibility result in mechanism design is known as the Gibbard-Satterthwaite theorem [10, 13].

Although a manipulation is guaranteed to exist (for reasonable rules), in order for the manipulating agent to use it, she must also be able to find it. Recent research has studied whether finding a manipulation can be made computationally hard, thereby erecting a computational barrier against manipulation. In early work $[2,1]$, it was shown that when the number of alternatives is not bounded, the second-order Copeland and STV rules are hard to manipulate, respectively. More recent research has studied how to modify other existing rules to make them hard to manipulate [3, 7]. Also, it has been shown that richer variants of the manipulation problem (manipulation by coalitions of weighted voters) can be hard even with a constant number of alternatives [5, 11].

However, all of these hardness results are worst-case results. That is, they suggest that any algorithm will require exponential time to solve some instances. However, this does not mean that there is no efficient algorithm that can find a manipulation for most instances. Indeed, several recent results suggest that finding manipulations is usually easy. Procaccia and Rosenschein have shown that, when the number of alternatives is a constant, manipulation of positional scoring rules is easy even with respect to "junta" distributions, which arguably focus on hard instances [12]. Conitzer and Sandholm have given some sufficient conditions under which manipulation is easy and argue that these conditions are usually satisfied in practice [4]. Zuckerman et al. have given manipulation algorithms with the property that if they fail to find a manipulation when one exists, then, if the manipulators are given some additional vote weights, the algorithm will succeed [14].

Following this line of research, a quantitative version of the GibbardSatterthwaite theorem was recently proved [9]. This theorem states that, when there are three alternatives, for any neutral voting rule 
that is far from any dictatorship, a random manipulation- that is, the true preferences and the strategic vote are all drawn i.i.d., uniformly at random - will succeed with a probability of $\Omega\left(\frac{1}{n}\right)$, where $n$ is the number of voters. This is perhaps one of the nicest approaches so far in trying to show that it is usually easy to find manipulations; unfortunately, it seems that the technique used in the proof of the theorem cannot be easily extended to four or more alternatives. In this paper, we obtain a similar result that does extend to four or more alternatives, albeit under different assumptions.

After recalling some basic definitions and notations in Section 2, we prove our main result in Section 3. Instead of considering neutral voting rules that are far from a dictatorship, our theorem applies to all voting rules that satisfy the following five conditions: 1 . homogeneity (if all the votes are multiplied by the same factor, the outcome does not change), 2. anonymity (the rule treats all voters equally), 3. non-imposition, 4. a canceling-out condition (if the set of all linear orders is added to the votes, then the outcome does not change), and 5. there exists a stable profile that is still stable after one given alternative is uniformly moved to different positions (and a profile is stable if slight perturbations do not change the winner). Our theorem states that for any fixed number of alternatives, if a voting rule satisfies the five conditions, then there exists a voter such that a random manipulation for that voter succeeds with a probability of $\Omega\left(\frac{1}{n}\right)$. Finally, in Section 5, we show that some common voting rules, including all positional scoring rules, STV, Copeland, maximin, and ranked pairs, satisfy the five conditions.

\section{PRELIMINARIES}

Let $\mathcal{C}=\left\{c_{1}, \ldots, c_{m}\right\}$ be the set of alternatives (or candidates). A linear order on $\mathcal{C}$ is a transitive, antisymmetric, and total relation on $\mathcal{C}$. The set of all linear orders on $\mathcal{C}$ is denoted by $L(\mathcal{C})$. An $n$-voter profile $P$ on $\mathcal{C}$ consists of $n$ linear orders on $\mathcal{C}$. That is, $P=\left(V_{1}, \ldots, V_{n}\right)$, where for every $i \leq n, V_{i} \in L(\mathcal{C})$. The set of all profiles on $\mathcal{C}$ is denoted by $P(\mathcal{C})$. In the remainder of the paper, $m$ denotes the number of alternatives and $n$ denotes the number of voters.

A voting rule $r$ is a function from the set of all profiles on $\mathcal{C}$ to $\mathcal{C}$, that is, $r: P(\mathcal{C}) \rightarrow \mathcal{C}$. The following are some common voting rules.

1. (Positional) scoring rules: Given a scoring vector $\vec{v}=(v(1), \ldots, v(m))$, for any vote $V \in L(\mathcal{C})$ and any $c \in \mathcal{C}$, let $s(V, c)=v(j)$, where $j$ is the rank of $c$ in $V$. For any profile $P=\left(V_{1}, \ldots, V_{n}\right)$, let $s(P, c)=\sum_{i=1}^{n} s\left(V_{i}, c\right)$. The rule will select $c \in \mathcal{C}$ so that $s(P, c)$ is maximized. Two examples of scoring rules are Borda, for which the scoring vector is $(m-1, m-2, \ldots, 0)$, and plurality, for which the scoring vector is $(1,0, \ldots, 0)$.

2. Copeland: For any two alternatives $c_{i}$ and $c_{j}$, we can simulate a pairwise election between them, by seeing how many votes prefer $c_{i}$ to $c_{j}$, and how many prefer $c_{j}$ to $c_{i}$. Then, an alternative receives one point for each win in a pairwise election. Typically, an alternative also receives half a point for each pairwise tie. The winner is the alternative who has the highest score.

3. STV: The election has $|\mathcal{C}|$ rounds. In each round, the alternative that gets the minimal plurality score drops out, and is removed from all of the votes (so that votes for this alternative transfer to another alternative in the next round). The last-remaining alternative is the winner.
4. Maximin: Let $N\left(c_{i}, c_{j}\right)$ denote the number of votes that rank $c_{i}$ ahead of $c_{j}$. The winner is the alternative $c$ that maximizes $\min \left\{N\left(c, c^{\prime}\right): c^{\prime} \in \mathcal{C}, c^{\prime} \neq c\right\}$.

5. Bucklin: An alternative c's Bucklin score is the smallest number $k$ such that more than half of the votes rank $c$ among the top $k$ alternatives. The winner is the alternative who has the smallest Bucklin score. (Sometimes, ties are broken by the number of votes that rank an alternative among the top $k$.)

6. Ranked pairs: This rule first creates an entire ranking of all the alternatives. $N\left(c_{i}, c_{j}\right)$ is defined as for the maximin rule. In each step, we will consider a pair of alternatives $c_{i}, c_{j}$ that we have not previously considered; specifically, we choose the remaining pair with the highest $N\left(c_{i}, c_{j}\right)$. We then fix the order $c_{i}>c_{j}$, unless this contradicts previous orders that we fixed (that is, it violates transitivity). We continue until we have considered all pairs of alternatives (hence we have a full ranking). The alternative at the top of the ranking wins.

6. Dictatorship: For each voter $i$, the rule where $i$ is the dictator always chooses the alternative at the top of $V_{i}$.

A manipulation for voter $i$ is a tuple $\left(V_{-i}, V_{i}, V_{i}^{\prime}\right)$, where $V_{-i}$ corresponds to the true preferences of all the voters except $i, V_{i}$ corresponds to the true preferences of voter $i$, and $V_{i}^{\prime}$ is the strategic vote of voter $i$. A manipulation is successful under voting rule $r$ if $r\left(V_{-i}, V_{i}^{\prime}\right) \succ_{V_{i}} r\left(V_{-i}, V_{i}\right)$. That is, if voter $i$ submits $V_{i}^{\prime}$ instead of $V_{i}$, this results in a winner that is more preferred by voter $i$. In this paper, $V_{-i}$ is drawn uniformly from $L(\mathcal{C})^{n-1}$, and $V_{i}$ and $V_{i}^{\prime}$ are drawn uniformly from $L(\mathcal{C})$; in this case, $\left(V_{-i}, V_{i}, V_{i}^{\prime}\right)$ is a random manipulation for voter $i$. Let $M_{i, m, n}(r)$ denote the probability that a random manipulation for voter $i$ is successful.

For two voting rules $f$ and $g$, let $\Delta_{m, n}(f, g)$ be the distance between two voting rules $f$ and $g$ w.r.t. $m$ alternatives and $n$ voters, defined as follows:

$$
\Delta_{m, n}(f, g)=\operatorname{Pr}_{P \in L(\mathcal{C})^{n}}(f(P) \neq g(P))
$$

Here, $P$ is drawn uniformly from $L(\mathcal{C})^{n}$. The following theorem (proved by Friedgut et al. [9]) states that when there are three alternatives, if the distance between a neutral voting rule $r$ and any dictatorship is at least $\epsilon>0$, then $\sum_{i} M_{i, 3, n}(r)$ is $\Omega\left(\epsilon^{2}\right)$.

Theorem 1 (Theorem 1 in [9]) When $m=3$, there exists a constant $C$ such that if there exists $\epsilon>0$ such that for any dictatorship dict, $\Delta_{3, n}(r$, dict $)>\epsilon$, then $\sum_{i=1}^{n} M_{i, 3, n}(r)>C \epsilon^{2}$.

This theorem implies that for any voting rule that is $\epsilon$-far away from any dictatorship for any number of voters, there exists a voter $i$ such that with a probability of $\Omega\left(\frac{1}{n}\right)$, a random manipulation for voter $i$ is successful.

\section{MAIN THEOREM}

In the remainder of this paper, we assume that all the distributions are uniform. For any set $S$, let $L(S)$ be all the linear orders over $S$. Given a linear order $V$ over $S$ and $S_{n}^{\prime} \subset S$, let $\left.V\right|_{S_{n}^{\prime}}$ be the restriction of $V$ to $S_{n}^{\prime}$. Similarly, let $\left.P\right|_{S_{n}^{\prime}}$ be the restriction of the profile $P$ to $S_{n}^{\prime}$. For any subset of agents $I \subseteq\{1, \ldots, n\}$ and any $n$-voter profile $P=\left\{V_{1}, \ldots, V_{n}\right\}$, let $P(I)=\left\{V_{i}: i \in I\right\}$. In this paper, we focus on anonymous rules (that is, rules that do not distinguish among the voters), so that we can represent a profile as a (multi)set rather than a vector. Let $i P$ be the profile that is obtained from $P$ by replacing each vote with $i$ copies of it. A rule $r$ is homogenous if for any $P$ and $i, r(P)=r(i P)$. 
Definition 1 Given a voting rule $r$ that satisfies anonymity and homogeneity, a profile $P$ is stable if there exists $\epsilon>0$ such that for any $i \in \mathbb{N}$ and any profile $P^{\prime}$ with $\left|P^{\prime}\right|<\epsilon i|P|$,

$$
r\left(i P \cup P^{\prime}\right)=r(P)
$$

We emphasize that stability is a property of a profile with respect to a rule, not just of a rule. If fractional votes are allowed, then a profile is stable (with respect to a rule) if there exists an $\epsilon$ such that if we add a set of votes whose size is at most $\epsilon$ times the size of the original set, then the winner does not change. However, we are interested in the case where fractional votes are not allowed. This is the reason that we need the $i$ in the definition.

Definition 2 Let us consider a social choice setting with $m$ alternatives and $n$ voters, where social choice rule $r$ is being used. Also suppose we draw $V_{1}, \ldots, V_{n}$ and $V_{i}^{\prime}$ uniformly at random. Then, for any $i \leq n$, let $M_{i, m, n}(r)$ be the probability that $r\left(V_{1}, \ldots, V_{i}^{\prime}, \ldots, V_{n}\right) \succ_{V_{i}} r\left(V_{1}, \ldots, V_{n}\right)$-that is, $V_{i}^{\prime}$ is a successful manipulation for $i$.

Let $C_{m}=\left\{c_{1}, \ldots, c_{m}\right\}$ be the set of alternatives.

Definition 3 Define the merging function $M: L\left(C_{m}\right)^{n} \times\{1, \ldots, m\}^{n} \rightarrow L\left(C_{m}\right)^{n}$ such that

$$
\left.M\left(\left(V_{1}, \ldots, V_{n}\right),\left(i_{1}, \ldots, i_{n}\right)\right)\right)=\left(M\left(V_{1}, i_{1}\right), \ldots, M\left(V_{n}, i_{n}\right)\right)
$$

where $M\left(V_{j}, i_{j}\right)$ is the vote that results from moving $c_{m}$ to the $i_{j}$ th position. (We note that we allow $M$ to be applied both to vectors and to individual elements.)

For example, $M\left(\left(c_{1} \succ c_{2} \succ c_{3}, c_{2} \succ c_{3} \succ c_{1}\right),(1,3)\right)=\left(c_{3} \succ\right.$ $\left.c_{1} \succ c_{2}, c_{2} \succ c_{1} \succ c_{3}\right)$.

We now define a function $D_{m}$ that maps any profile $P$ of $n$ votes to another profile $D_{m}(P)$ such that the position of $c_{m}$ is uniformly redistributed, while keeping the restriction of the profile to $C_{m-1}=\left\{c_{1}, \ldots, c_{m-1}\right\}$ unchanged. The size of the resulting profile is still $n$; however, some of the votes in the resulting profile are fractional. The set $L\left(C_{m}\right)$ of linear orders of the alternatives has $m$ ! elements; let $l_{i}$ denote the $i$ th linear order $(1 \leq i \leq m$ !). Then, any profile $P$ can be written as $\sum_{i=1}^{m !} p_{i} l_{i}$, for some $p_{i}$ that indicate how many times each linear order occurs. (This is making use of the fact that we only consider anonymous rules.)

Definition 4 For any profile $P=\sum_{i=1}^{m !} p_{i} l_{i}$, let

$$
D_{m}(P)=\sum_{i=1}^{m !} \sum_{j=1}^{m} \frac{p_{i}}{m} M\left(l_{i}, j\right)
$$

Definition 5 Let $P^{\prime}=L\left(C_{m}\right)$ be the profile of all linear orders, each appearing exactly once. We say a rule $r$ satisfies canceling out if for any profile $P, r\left(P \cup P^{\prime}\right)=r(P)$.

We are now ready to present our main result. This result states that any rule that satisfies certain conditions is, in a sense, frequently manipulable. (The precise definition of "frequently manipulable" is the same as that used by Friedgut et al.)

Theorem 2 Suppose that a voting rule $r$ that chooses an alternative from $C_{m}=\left\{c_{1}, \ldots, c_{m}\right\}$ satisfies the following properties:

\section{Homogeneity.}

2. Anonymity.

3. Non-imposition, which means that for any $n$, any alternative $c_{i}$, there exists a profile $P$ of $n$ votes such that $r(P)=c_{i}$.

\section{Canceling out.}

5. There exists a profile $P$ such that: (a) $P$ and $D_{m}(P)$ are both stable, (b) $r(P)=c_{1}$, and $(c) r\left(D_{m}(P)\right)=c_{2}$.

Then, there exists $\epsilon>0$ such that for any $n \in \mathbb{N}, \sum_{i=1}^{n} M_{i, m, n}(r)>$ $\epsilon$. Here, $\epsilon$ does not depend on $n$.

We note that Theorem 1 [9] relates the frequency of manipulation under any neutral voting rule to the distance between the rule and dictatorships, when the number of alternatives is three. In contrast, the main theorem in this paper (Theorem 2) applies to any number of alternatives, and implies that the frequency of manipulation under any voting rule satisfying the five conditions in Theorem 2 is non-negligible. The conditions in Theorem 1 and Theorem 2 are not comparable. Theorem 1 assumes neutrality, and Theorem 2 does not. The assumption of anonymity in Theorem 2 is related to the dependence on the distance to the dictatorships in Theorem 1. Admittedly, Condition 5 in Theorem 2 is less natural than all the other conditions. In Section 5, we will show that many of the common rules satisfy the five conditions (including Condition 5) in Theorem 2.

\section{PROOF OF MAIN THEOREM}

The proof of our main theorem follows the line of the proof of Theorem 1 in [9]. First, for any voting rule $r$, we define the quantity $M_{n}^{m-1}(r)$ to be the probability that the winners under $r$ for two randomly drawn profiles are the same, given that the restrictions of the two profiles to $C_{m-1}=\left\{c_{1}, \ldots, c_{m-1}\right\}$ are the same. Second, we prove that, for any voting rule $r$ satisfying the five conditions in Theorem 2 and any fixed number of alternatives $m, M_{n}^{m-1}(r)$ is non-negligible, meaning that it is always strictly larger than some positive constant (Lemma 2). Third, we bound the frequency of manipulation below by $M_{n}^{m-1}(r)$.

The first and third steps are natural extensions of their counterparts in [9]. However, the technique used to prove the second part is quite different. For this part, we bound the probability that a randomly drawn profile satisfies the following two conditions below by a constant. 1 . The profile is approximately multiple copies of the profile $P$ in Condition 5. 2. After "shifting" $c_{m}$ in each vote in the profile to a random position in the vote, the new profile is approximately multiple copies of $D_{m}(P)$ with a non-negligible probability.

We first present a basic result in probability theory that we will need in the proof. The result is a corollary of a known multivariate version of the central limit theorem; this multivariate version can be found in Dudley [6] ${ }^{1}$.

Lemma 1 Suppose each random variable $X_{i}(1 \leq i \leq n)$ is uniformly (i.i.d.) drawn from $\left\{x_{1}, \ldots, x_{m}\right\}$. Let $P=\left(X_{1}, \ldots, X_{n}\right)$, $t_{i}(P)$ be the number of times $x_{i}$ occurs in $P$. Then, for any $q_{1}, \ldots, q_{m} \in$ $\mathbb{R}$ such that $q_{1}+\ldots+q_{m}=0$ and any $\epsilon>0$, there exists $\delta_{q_{1}, \ldots, q_{m}}>0$ (which does not depend on $n$ ) such that

$$
\lim _{n \rightarrow \infty} \operatorname{Pr}\left(\bigcap_{i=1}^{m}\left(\left|t_{i}(P)-\frac{n}{m}-q_{i} \sqrt{n}\right|<\epsilon \sqrt{n}\right)\right)>\delta_{q_{1}, \ldots, q_{m}}
$$

Proof of Lemma 1: We first note that

$$
\begin{aligned}
& \operatorname{Pr}\left(\bigcap_{i=1}^{m}\left(\left|t_{i}(P)-\frac{n}{m}-q_{i} \sqrt{n}\right|<\epsilon \sqrt{n}\right)\right) \\
\geq & \left.\operatorname{Pr}\left(\bigcap_{i=1}^{m-1}\left|t_{i}(P)-\frac{n}{m}-q_{i} \sqrt{n}\right|<\frac{\epsilon}{m} \sqrt{n}\right)\right)
\end{aligned}
$$

\footnotetext{
${ }^{1}$ We thank Sayan Mukherjee for referring us to this result.
} 
This inequality holds because $\sum_{i=1}^{m}\left(t_{i}(P)-\frac{n}{m}-q_{i} \sqrt{n}\right)=0$, which means that if $\left|t_{i}(P)-\frac{n}{m}-q_{i} \sqrt{n}\right|$ is small for all $i=$ $1, \ldots, m-1$, then $\left|t_{m}(P)-\frac{n}{m}-q_{m} \sqrt{n}\right|=\mid \sum_{i=1}^{m-1}\left(t_{i}(P)-\frac{n}{m}-\right.$ $\left.q_{i} \sqrt{n}\right) \mid$ is also small. For each $i \leq n$, let $\mathscr{X}_{i}$ be drawn uniformly and i.i.d. from the following set of $m-1$-dimensional vectors:

$\{(1, \ldots, 0),(0,1, \ldots, 0), \ldots,(0, \ldots, 1),(0, \ldots, 0)\}$. By definition, $\mathscr{X}_{i}$ is a vector-valued random variable in $\mathbb{R}^{m-1}$. Let $\mathscr{P}=$ $\sum_{i=1}^{n} \mathscr{X}_{i}$. Then

$$
\begin{aligned}
& \left.\operatorname{Pr}\left(\bigcap_{i=1}^{m-1}\left|t_{i}(P)-\frac{n}{m}-q_{i} \sqrt{n}\right|<\frac{\epsilon}{m} \sqrt{n}\right)\right) \\
= & \operatorname{Pr}\left(\bigcap_{i=1}^{m-1} \frac{n}{m}+q_{i} \sqrt{n}-\frac{\epsilon}{m} \sqrt{n}<\mathscr{P}(i)<\frac{n}{m}+q_{i} \sqrt{n}+\frac{\epsilon}{m} \sqrt{n}\right) \\
= & \operatorname{Pr}\left(\bigcap_{i=1}^{m-1} q_{i}-\frac{\epsilon}{m}<\frac{\mathscr{P}(i)-\frac{n}{m}}{\sqrt{n}}<q_{i}+\frac{\epsilon}{m}\right)
\end{aligned}
$$

where $\mathscr{P}(i)$ is the $i$-th component of $\mathscr{P}$. It is easy to check that the covariance matrix of $\mathscr{X}$ is positive definite, which means its rank is $m-1$. Therefore from Theorem 9.5.6 (the central limit theorem) and Theorem 9.5.7 in [6], when $n$ goes to infinity, $\frac{\mathscr{P}-\frac{n}{m}}{\sqrt{n}}$ converges to a multivariate normal distribution in $\mathbb{R}^{m-1}$,

$$
\begin{aligned}
& N(0, \Sigma)=f_{X}\left(x_{1}, \ldots, x_{m-1}\right) \\
= & \frac{1}{(2 \pi)^{(m-1) / 2}|\Sigma|^{1 / 2}} \exp \left(-\frac{1}{2} x^{\top} \Sigma^{-1} x\right)
\end{aligned}
$$

Similarly to the proof of Theorem 9.3.6 in [6], because the probability density function of a multivariate normal distribution is continuous, we can prove that when $n$ goes to infinity,

$$
\begin{aligned}
& \operatorname{Pr}\left(\bigcap_{i=1}^{m-1} q_{i}-\frac{\epsilon}{m}<\frac{\mathscr{P}(i)-\frac{n}{m}}{\sqrt{n}}<q_{i}+\frac{\epsilon}{m}\right) \\
= & \int_{q_{1}-\frac{\epsilon}{m}}^{q_{1}+\frac{\epsilon}{m}} \cdots \int_{q_{m-1}-\frac{\epsilon}{m}}^{q_{m-1}+\frac{\epsilon}{m}} N(0, \Sigma) d x_{m-1} \ldots d x_{1}
\end{aligned}
$$

Since $\int_{q_{1}-\frac{\epsilon}{m}}^{q_{1}+\frac{\epsilon}{m}} \cdots \int_{q_{m-1}-\frac{\epsilon}{m}}^{q_{m-1}+\frac{\epsilon}{m}} N(0, \Sigma) d x_{m-1} \ldots d x_{1}>0$, there exists $\delta_{q_{1}, \ldots, q_{m}}>0$ such that

$$
\left.\lim _{n \rightarrow \infty} \operatorname{Pr}\left(\bigcap_{i=1}^{m}\left|t_{i}(P)-\frac{n}{m}-q_{i} \sqrt{n}\right|<\epsilon \sqrt{n}\right)\right)>\delta_{q_{1}, \ldots, q_{m}}
$$

This lemma tells us that given "displacements" $q_{1}, \ldots, q_{m}$, there is some $\delta_{q_{1}, \ldots, q_{m}}$ such that when $n$ goes to infinity, the probability that for each $i, t_{i}(P)$ (the number of times $x_{i}$ occurs in $P$ ) is within $\epsilon \sqrt{n}$ of its expectation $n / m$ plus the displacement $q_{i} \sqrt{n}$, is at least $\delta_{q_{1}, \ldots, q_{m}}$.

We now define a quantity $M_{n}^{m-1}(r)$. We recall that for any profile $P$ over the set of alternatives $C_{m}=\left\{c_{1}, \ldots, c_{m}\right\},\left.P\right|_{C_{m-1}}$ is the profile obtained by removing $c_{m}$ from each vote in $P$.

Definition 6 Suppose we draw profiles $P_{1}, P_{2}$ uniformly at random. Let $M_{n}^{m-1}(r)$ denote the conditional probability that $r\left(P_{1}\right)=$ $c_{1}$ and $r\left(P_{2}\right)=c_{2}$, given that $\left.P_{1}\right|_{C_{m-1}}=\left.P_{2}\right|_{C_{m-1}}$.

We will first show that when $n$ goes to infinity, $M_{n}^{m-1}(r)$ is bounded below by a quantity that does not depend on $n$. Then, we will relate $M_{n}^{m-1}(r)$ to $\sum_{i=1}^{n} M_{i, m, n}(r)$, which will show that the latter is bounded below by a quantity that does not depend on $n$.
Lemma 2 If $r$ satisfies all five conditions in Theorem 2, then there exists $n^{\prime} \in \mathbb{N}$ and $\delta>0$ (which does not depend on $n$ ) such that for any $n>n^{\prime}, M_{n}^{m-1}(r)>\delta$.

Proof of Lemma 2: Suppose $r$ satisfies all five conditions. Let the stable profile in Condition 5. of Theorem 2 be $P_{s}=q_{1} l_{1}+$ $\ldots+q_{m} l_{m !}$, and let $\epsilon>0$ be such that $P_{s}$ and $D_{m}\left(P_{s}\right)$ are both stable with respect to $\epsilon$. Let $\bar{q}=\frac{\sum_{i=1}^{m !} q_{i}}{m !}$, and $q_{i}^{\prime}=q_{i}-\bar{q}$. Also let $S_{n}$ denote the profiles $P$ of $n$ voters such that the number of occurrences $t_{i}(P)$ of each vote $l_{i}$ in $P$ is within $\frac{\epsilon}{4 m !} \sqrt{n}$ of $\frac{n}{m !}+q_{i}^{\prime} \sqrt{n}$, that is,

$S_{n}=\left\{P\right.$ : for every $\left.i \leq m !,\left|t_{i}(P)-\frac{n}{m !}-q_{i} \sqrt{n}\right|<\frac{\epsilon}{4 m !} \sqrt{n}\right\}$

By Lemma 1 we know that there exists $\delta_{q 1, \ldots, q_{m} \text { ! }}$ such that

$$
\lim _{n \rightarrow \infty} \operatorname{Pr}\left(P \in S_{n}\right)>\delta_{q_{1}, \ldots, q_{m !}}
$$

We first make the following claim.

Claim 1 For any $P \in S_{n}, r(P)=c_{1}$.

Proof of Claim 1: Since for any profile $P \in S_{n}$, any $i \leq m$ !, the number of $l_{i}$ in $P$ is more than $\frac{n}{m !}-\bar{q} \sqrt{n}-\frac{\epsilon}{4 m !} \sqrt{n}$, we can decompose $P$ into the following three parts.

1. The "canceling-out" part: $C P(P) \subset\{1, \ldots, n\}$, such that $P(C P(P))$ is $\left(\frac{n}{m !}-\left(\bar{q}+\frac{\epsilon}{4 m !}\right) \sqrt{n}\right)\left(l_{1}+\ldots+l_{m !}\right)$.

2. The "main" part $M P(P)$ : from the definition of the $S$, in $P(\overline{C P(P)})$ we can find $P(M P(P))=\sqrt{n}\left(\sum_{i=1}^{m !} q_{i} l_{i}\right)$.

3. The "negligible" part $N P(P)$ : all the remaining $\frac{\epsilon}{4 m !} \sqrt{n}$. $m !=\frac{\epsilon}{4} \sqrt{n}$ votes.

Therefore

$$
\begin{array}{rlr} 
& r(P)=r(P(M P(P))+P(N P(P))) & \text { canceling out } \\
= & r\left(\sqrt{n} P_{s}+N P(P)\right) & \text { anonymity of } r \\
= & \left.r\left(\sqrt{n} P_{s}\right)\right) & \text { stability } \\
= & r\left(P_{s}\right) & \text { homogeneity } \\
= & c_{1} &
\end{array}
$$

(End of the proof of Claim 1.)

For any pair $\left(P_{1}, P_{2}\right)$ such that $\left.P_{1}\right|_{C_{m-1}}=\left.P_{2}\right|_{C_{m-1}}$, we can represent this pair as $\left(P_{1}, K_{2}\right)$ where $K_{2} \in\{1, \ldots, m\}^{n}$. Here, the $i$ th component of $K_{2}$ indicates the position of $c_{m}$ in the $i$ th vote in $P_{2}$. We remember that for the earlier-defined merging function $M, M\left(P_{1}, K_{2}\right)=P_{2}$.

For any $P_{1} \in S$, we further decompose the canceling-out part and the main part as follows.

1. For each $l_{i}$ and $i \leq m$ !, let $A\left(P_{1}, l_{i}\right)$ denote the subscripts of all the votes in the canceling-out part $C P\left(P_{1}\right)$ that are equal to $l_{i}$, that is,

$$
A\left(P_{1}, l_{i}\right)=\left\{j \leq n: j \in C P\left(P_{1}\right) \text { and } P_{1}(j)=l_{i}\right\}
$$

2. For each $l_{i}, i \leq m$ !, let $B\left(P_{1}, l_{i}\right)$ denote the subscripts of all the votes in the main part $M P\left(P_{1}\right)$ that are equal to $l_{i}$, that is,

$$
B\left(P_{1}, l_{i}\right)=\left\{j \leq n: j \in M P\left(P_{1}\right) \text { and } P_{1}(j)=l_{i}\right\}
$$


We note that $\bigcup_{i=1}^{m !} A\left(P_{1}, l_{i}\right)=C P\left(P_{1}\right)$ and $\bigcup_{i=1}^{m !} B\left(P_{1}, l_{i}\right)=$ $M P\left(P_{1}\right)$.

What we will do next is to define a function $S_{n}^{\prime}: S_{n} \rightarrow 2^{\{1, \ldots, m\}^{n}}$ That is, for every profile $P_{1} \in S_{n}$, and every $K_{2} \in\{1, \ldots, m\}^{n}$, the function $S_{n}^{\prime}$ determines whether or not $K_{2} \in S_{n}^{\prime}\left(P_{1}\right)$. We want this function to have the following properties:

1. There exists some $n^{\prime}$ and some $\delta^{\prime}>0$ such that for any $n>n^{\prime}, \frac{\left|S_{n}^{\prime}\left(P_{1}\right)\right|}{m^{n}}>\delta^{\prime}$ (that is, if we are drawing an element uniformly at random from $\{1, \ldots, m\}^{n}$, then with a probability of at least $\delta^{\prime}$ the element we draw is in $S_{n}^{\prime}\left(P_{1}\right)$ ).

2. For every $K_{2} \in S_{n}^{\prime}\left(P_{1}\right), r\left(M\left(P_{1}, K_{2}\right)\right)=c_{2}$.

Again we make use of Lemma 1. This time we apply the lemma to the $K_{2}$ component. We recall that $K_{2}$ takes values in $\{1, \ldots, m\}^{n}$. We require that for every $P_{1} \in S_{n}$, for every $K_{2} \in S_{n}^{\prime}\left(P_{1}\right)$, for every $l_{i}$, each $j \in\{1, \ldots, m\}$ occurs approximately the same number of times in $K_{2}\left(A\left(P_{1}, l_{i}\right)\right)$, and each $j \in\{1, \ldots, m\}$ occurs approximately the same number of times in $K_{2}\left(B\left(P_{1}, l_{i}\right)\right.$ ). (For any $C \subseteq\{1, \ldots, n\}, K_{2}(C)$ is the subvector of $K_{2}$ consisting of the components in $C$.)

We will first define $S_{n, A, l_{i}}^{\prime}\left(P_{1}\right) \subseteq\{1, \ldots, m\}^{A\left(P_{1}, l_{i}\right)}$ and $S_{n, B, l_{i}}^{\prime}\left(P_{1}\right) \subseteq\{1, \ldots, m\}^{B\left(P_{1}, l_{i}\right)}$, which are the projections of the $K_{2} \in S_{n}^{\prime}\left(P_{1}\right)$ onto the components in $A\left(P_{1}, l_{i}\right)$ and $B\left(P_{1}, l_{i}\right)$, respectively. The definition is as follows: $K_{2}^{\prime}$ is in $S_{n, A, l_{i}}^{\prime}\left(P_{1}\right)$ if and only if for any $j \leq m$,

$$
\left|t_{j}\left(K_{2}^{\prime}\right)-\frac{\left|A\left(P_{1}, l_{i}\right)\right|}{m}\right|<\frac{\epsilon \sqrt{\left|A\left(P_{1}, l_{i}\right)\right|}}{4 m ! \cdot m}
$$

$K_{2}^{\prime}$ is in $S_{n, B, l_{i}}^{\prime}\left(P_{1}\right)$ if and only if for any $j \leq m$,

$$
\left|t_{j}\left(K_{2}^{\prime}\right)-\frac{\left|B\left(P_{1}, l_{i}\right)\right|}{m}\right|<\frac{\epsilon \sqrt{\left|B\left(P_{1}, l_{i}\right)\right|}}{4 m \cdot \sqrt{q_{\max }}},
$$

where $q_{\max }=\max \left\{q_{1}, \ldots, q_{m !}\right\}$.

We are now ready to define $S_{n}^{\prime}$. We define $S_{n}^{\prime}\left(P_{1}\right)$ to be the set of profiles such that $K_{2} \in S_{n}^{\prime}\left(P_{1}\right)$ if and only if for any $i \leq m$ !, each of $\{1, \ldots, m\}$ occurs approximately equally many times in $K_{2}\left(A\left(P_{1}, l_{i}\right)\right)$ and $K_{2}\left(B\left(P_{1}, l_{i}\right)\right)$. That is:

$S_{n}^{\prime}\left(P_{1}\right)=\left\{K_{2} \in\{1, \ldots, m\}^{n}\right.$ : for every $i \leq m$ !,

$K_{2}\left(A\left(P_{1}, l_{i}\right)\right) \in S_{n, A, l_{i}}^{\prime}\left(P_{1}\right)$ and $\left.K_{2}\left(B\left(P_{1}, l_{i}\right)\right) \in S_{n, B, l_{i}}^{\prime}\left(P_{1}\right)\right\}$

We note that we are ignoring the the $K_{2}$ component of the negligible part $N P\left(P_{1}\right)$.

We will now prove that properties 1 and 2 above hold. For property 1 , since each element of $K_{2}$ is drawn independently, $K_{2}$ can be determined in the following $2 m$ ! +1 steps: first draw the $K_{2}$ component of $A\left(P_{1}, l_{1}\right)$, then that of $A\left(P_{1}, l_{2}\right)$, and so on, until $B\left(P_{1}, l_{m !}\right)$, in the last step draw all the remaining components (the ones in the negligible part) arbitrarily. By Lemma 1, there exists $\delta_{0, \ldots, 0}$ such that when $n$ goes to infinity, for the step concerning $A\left(P_{1}, l_{i}\right)$, the probability that its $K_{2}$ component is in $S_{n, A, l_{i}}^{\prime}\left(P_{1}\right)$ is larger than $\delta_{0, \ldots, 0}$ (and similarly for the $B\left(P_{1}, l_{i}\right)$ ). Hence,

$$
\lim _{n \rightarrow \infty} \operatorname{Pr}\left(K_{2} \in S_{n}^{\prime}\left(P_{1}\right)\right)>\left(\delta_{0, \ldots, 0}\right)^{2 m !}
$$
that

Therefore there exists a $\delta>0$ that does not depend on $n$ such

$$
\lim _{n \rightarrow \infty} \operatorname{Pr}\left(\left(P_{1}, S_{n}^{\prime}\left(P_{1}\right)\right): P_{1} \in S_{n}\right)>\delta
$$

Specifically, $\delta=\delta_{q_{1}, \ldots q_{m !}} \cdot\left(\delta_{0, \ldots, 0}\right)^{2 m !}$. We now make the following claim.
Claim 2 For any $P_{1} \in S_{n}$, any $K_{2} \in S_{n}^{\prime}\left(P_{1}\right), r\left(M\left(P_{1}, K_{2}\right)\right)=$ $c_{2}$.

Proof of Claim 2: We first prove that for any $K_{2} \in S_{n}^{\prime}\left(P_{1}\right)$, $M\left(P_{1}, K_{2}\right)\left(C P\left(P_{1}\right)\right)$ (that is, the projection of the profile resulting from the merge operator onto the elements that originally canceled out) still approximately cancels out. Then, we prove that $M\left(P_{1}, K_{2}\right)\left(M P\left(P_{1}\right)\right)$ (the projection of the new profile onto the elements that were originally in the main part) consists approximately of multiple copies of $D_{m}\left(P_{s}\right)$. Then, by a similar argument as the one in Claim 1, it follows that $r\left(M\left(P_{1}, K_{2}\right)\right)=c_{2}$.

First, for any $l_{i}$, the number of votes $l_{i}$ in $M\left(P_{1}, K_{2}\right)\left(C P\left(P_{1}\right)\right)$ can be bounded below as follows:

$$
t_{i}\left(M\left(P_{1}, K_{2}\right)\left(C P\left(P_{1}\right)\right)\right)>t_{i}\left(C P\left(P_{1}\right)\right)-\frac{\epsilon \sqrt{n}}{4 m !}
$$

This is because for any $i \leq m$ !, the votes $l_{i}$ in $M\left(P_{1}, K_{2}\right)\left(C P\left(P_{1}\right)\right)$ result from $m$ sets in the original profile: if we let $l_{i_{j}}=M\left(l_{i}, j\right)$ be the order that agrees with $l_{i}$ on $C_{m-1}$ but places $c_{m}$ in the $j$ th position, then for each $j$, votes $l_{i}$ in the new profile result from the votes in $P_{1}\left(A\left(P_{1}, l_{i_{j}}\right)\right)$ in the original profile. For example, if $l_{i}=$ $c_{1} \succ \ldots \succ c_{m}$, then $l_{i_{1}}=c_{m} \succ c_{1} \succ \ldots \succ c_{m-1}, l_{i_{2}}=c_{1} \succ$ $c_{m} \succ c_{2} \succ \ldots \succ c_{m-1}, \ldots, l_{i_{m}}=l_{i}=c_{1} \succ \ldots \succ c_{m}$. Because $l_{i}$ occurs in $\left.M\left(P_{1}, K_{2}\right)\left(A\left(P_{1}, l_{i_{j}}\right)\right)\right)$ at least $\frac{\left|A\left(P_{1}, l_{i_{j}}\right)\right|}{m}-$ $\frac{\epsilon \sqrt{\left|A\left(P_{1}, l_{i_{j}}\right)\right|}}{4 m ! \cdot m}$ times by the definition of $S_{n, A, l_{i_{j}}}^{\prime}\left(P_{1}\right)$, and because $\left|A\left(P_{1}, l_{i_{j}}\right)\right|<n$, we have $\frac{\epsilon \sqrt{\left|A\left(P_{1}, l_{i_{j}}\right)\right|}}{4 m ! \cdot m}<\frac{\epsilon \sqrt{n}}{4 m ! \cdot m}$, which means

$$
\begin{aligned}
& t_{i}\left(M\left(P_{1}, K_{2}\right)\left(C P\left(P_{1}\right)\right)\right) \\
> & m \cdot\left(\frac{t_{i}\left(C P\left(P_{1}\right)\right)}{m}-\frac{\epsilon \sqrt{n}}{4 m ! \cdot m}\right) \\
= & t_{i}\left(C P\left(P_{1}\right)\right)-\frac{\epsilon \sqrt{n}}{4 m !}
\end{aligned}
$$

So, we can construct a new canceling-out part in $M\left(P_{1}, K_{2}\right)$ that contains most of the old canceling-out part $C P\left(P_{1}\right)$. That is, there exists $\operatorname{NCP}\left(P_{1}, K_{2}\right) \subseteq C P\left(P_{1}\right)$ such that

$$
\begin{aligned}
& M\left(P_{1}, K_{2}\right)\left(N C P\left(P_{1}, K_{2}\right)\right) \\
= & \left(\frac{n}{m !}-\left(\bar{q}+2 \cdot \frac{\epsilon}{4 m !}\right) \sqrt{n}\right)\left(V_{1}+\ldots+V_{m !}\right)
\end{aligned}
$$

The part of the old canceling-out part that is not in the new cancelingout part will turn out to be negligible.

Second, the number of times that any order $l_{i}$ occurs in $M\left(P_{1}, K_{2}\right)\left(M P\left(P_{1}\right)\right)$ can be bounded below as follows:

$$
t_{i}\left(M\left(P_{1}, K_{2}\right)\left(M P\left(P_{1}\right)\right)\right)>\sqrt{n} t_{i}\left(D_{m}\left(P_{s}\right)\right)-\frac{\epsilon \sqrt{n}}{4}
$$

This is true by a similar argument as before: for any $i \leq m$ !, the votes $l_{i}$ in $M\left(P_{1}, K_{2}\right)\left(M P\left(P_{1}\right)\right)$ result from $m$ sets in the original profile: for each $j$, votes $l_{i}$ in the new profile result from the votes 
in $P_{1}\left(B\left(P_{1}, l_{i_{j}}\right)\right)$ in the original profile. Therefore

$$
\begin{aligned}
& t_{i}\left(M\left(P_{1}, K_{2}\right)\left(M P\left(P_{1}\right)\right)\right) \\
> & \sum_{j=1}^{m}\left(\frac{q_{i_{j}} \sqrt{n}}{m}-\frac{\epsilon \sqrt{\left|B\left(P_{1}, l_{i_{j}}\right)\right|}}{4 m \cdot \sqrt{q_{\max }}}\right) \\
= & \sum_{j=1}^{m}\left(\frac{q_{i_{j}} \sqrt{n}}{m}-\frac{\epsilon \sqrt{q_{j_{i}} \sqrt{n}}}{4 m \cdot \sqrt{q_{\max }}}\right) \\
> & \sum_{j=1}^{m}\left(\frac{q_{i_{j}} \sqrt{n}}{m}-\frac{\epsilon \sqrt{q_{\max } \sqrt{n}}}{4 m \cdot \sqrt{q_{\max }}}\right) \\
= & \sum_{j=1}^{m} \frac{q_{i_{j}} \sqrt{n}}{m}-m \cdot \frac{\epsilon \sqrt[4]{n}}{4 m} \\
> & \sqrt{n} t_{i}\left(D_{m}\left(P_{s}\right)\right)-\frac{\epsilon \sqrt{n}}{4}
\end{aligned}
$$

The first equality follows from the fact that

$M P\left(P_{1}\right)=\sqrt{n} \sum_{i=1}^{m !} q_{i} l_{i}$, which means that $B\left(P_{1}, l_{i_{j}}\right)=q_{i_{j}} \sqrt{n}$. The last inequality follows from the fact that $\sqrt{n}>\sqrt[4]{n}$.

So, we can construct a new main part in $M\left(P_{1}, K_{2}\right)$ that contains most of the old main part $M P\left(P_{1}\right)$. That is, there exists $N M P\left(P_{1}, K_{2}\right) \subseteq M P\left(P_{1}\right)$ such that

$$
M\left(P_{1}, K_{2}\right)\left(N M P\left(P_{1}, K_{2}\right)\right)=\sqrt{n} D_{m}\left(P_{s}\right)-\frac{\epsilon \sqrt{n}}{4} \sum_{i=1}^{m !} l_{i}
$$

We let $N N P\left(P_{1}, K_{2}\right)$ denote the set of all the remaining votesconsisting of the votes from the old canceling-out part that are not in the new canceling-out part, the votes from the old main part that are not in the new main part, and the votes from the old negligible part. We know $\left|N N P\left(P_{1}, K_{2}\right)\right| \leq \frac{3 \epsilon \sqrt{n}}{4}<\epsilon \sqrt{n}$. By definition $N C P\left(P_{1}, K_{2}\right) \cup N M P\left(P_{1}, K_{2}\right) \cup N N P\left(P_{1}, K_{2}\right)=\{1, \ldots, n\}$. It follows that:

$$
\begin{aligned}
& r\left(M\left(P_{1}, K_{2}\right)\right) \\
= & r\left(\frac{\epsilon \sqrt{n}}{4} \sum_{i=1}^{m !} l_{i}+M\left(P_{1}, K_{2}\right)\left(N M P\left(P_{1}, K_{2}\right)\right)\right. \\
& \left.+M\left(P_{1}, K_{2}\right)\left(N N P\left(P_{1}, K_{2}\right)\right)\right) \\
= & r\left(\sqrt{n} D_{m}\left(P_{s}\right)+M\left(P_{1}, K_{2}\right)\left(N N P\left(P_{1}, K_{2}\right)\right)\right) \\
= & r\left(\sqrt{n} D_{m}\left(P_{s}\right)\right) \\
= & r\left(P_{s}\right) \\
= & c_{2}
\end{aligned}
$$

canceling out anonymity of $r$ homogeneity

(End of the proof of Claim 2.)

To conclude, there exists a $\delta>0$ which does not depend on $n$ such that

$$
\lim _{n \rightarrow \infty} \operatorname{Pr}\left(\left(P_{1}, S_{n}^{\prime}\left(P_{1}\right)\right): P_{1} \in S_{n}\right)>\delta
$$

and for any $P_{1} \in S_{n}$, any $K_{2} \in S_{n}^{\prime}\left(P_{1}\right), r\left(P_{1}\right)=c_{1}$ and $r\left(M\left(P_{1}, K_{2}\right)\right)=c_{2}$. So there exists $n^{\prime} \in \mathbb{N}$ s.t. when $n>n^{\prime}$, $\operatorname{Pr}\left(\left(P_{1}, S_{n}^{\prime}\left(P_{1}\right)\right): P_{1} \in S_{n}\right)>\frac{\delta}{2}$. Notice that for any $n \in \mathbb{N}$, $M_{n}^{m-1}(r) \geq \operatorname{Pr}\left(\left(P_{1}, S_{n}^{\prime}\left(P_{1}\right)\right): P_{1} \in S_{n}\right)$, we know that for any $n \geq n^{\prime}$,

$$
M_{n}^{m-1}(r) \geq \operatorname{Pr}\left(\left(P_{1}, S_{n}^{\prime}\left(P_{1}\right)\right): P_{1} \in S_{n}\right)>\frac{\delta}{2}
$$

(End of the proof for Lemma 3.)
We now obtain a lower bound on $\sum_{i=1}^{n} M_{i, m, n}(r)$ that depends on $M_{n}^{m-1}(r)$.

Lemma $3 \sum_{i=1}^{n} M_{i, m, n}(r)>\frac{1}{m !} M_{n}^{m-1}(r)$.

Proof of Lemma 3: This is an extension of Lemma 3 in [9]. For each $z^{m-1} \in L\left(C_{m-1}\right)^{n}$, let $A\left(z^{m-1}\right)$ and $B\left(z^{m-1}\right)$ be two subsets of $\{1, \ldots, m\}^{n}$ defined as follows:

$$
\begin{aligned}
& A\left(z^{m-1}\right)=\left\{y \in\{1, \ldots, m\}^{n}: r\left(M\left(z^{m-1}, y\right)\right)=c_{1}\right\} \\
& B\left(z^{m-1}\right)=\left\{y \in\{1, \ldots, m\}^{n}: r\left(M\left(z^{m-1}, y\right)\right)=c_{2}\right\}
\end{aligned}
$$

That is, $A\left(z^{m-1}\right)\left(B\left(z^{m-1}\right)\right)$ consists of the vectors of positions such that if we extend $z^{m-1}$ to include $c_{m}$ in those positions, then $c_{1}\left(c_{2}\right)$ wins. First we show that

$$
M_{n}^{m-1}(r)=E_{x}\left[\frac{\left|A\left(\left.x\right|_{C_{m-1}}\right)\right|}{m^{n}} \cdot \frac{\left|B\left(\left.x\right|_{C_{m-1}}\right)\right|}{m^{n}}\right]
$$

(here, $x$ is a vector of $n$ votes drawn uniformly at random), as follows:

$$
\begin{aligned}
& M_{n}^{m-1}(r) \\
= & \frac{1}{((m-1) !)^{n} \cdot m^{2 n}} \sum_{x^{m-1} \in L\left(C_{m-1}\right)^{n}} \mid\left\{(a, b): a, b \in\{1, \ldots, m\}^{n},\right. \\
& \left.r\left(M\left(x^{m-1}, a\right)\right)=c_{1}, r\left(M\left(x^{m-1}, b\right)\right)=c_{2}\right\} \mid \\
= & \frac{1}{((m-1) !)^{n} \cdot m^{2 n}} \sum_{x^{m-1} \in L\left(C_{m-1}\right)^{n}}\left|A\left(x^{m-1}\right)\right| \cdot\left|B\left(x^{m-1}\right)\right|
\end{aligned}
$$

Now, we have that

$$
\begin{aligned}
& \frac{1}{((m-1) !)^{n} \cdot m^{2 n}} \sum_{x^{m-1} \in L\left(C_{m-1}\right)^{n}}\left|A\left(x^{m-1}\right)\right| \cdot\left|B\left(x^{m-1}\right)\right| \\
= & \frac{1}{((m-1) !)^{n} \cdot m^{3 n}} \sum_{x \in L\left(C_{m}\right)^{n}}\left|A\left(\left.x\right|_{C_{m-1}}\right)\right| \cdot\left|B\left(\left.x\right|_{C_{m-1}}\right)\right|
\end{aligned}
$$

because for each $x^{m-1}$, there are $m^{n}$ different vectors $x \in L\left(C_{m}\right)^{n}$ that, when restricted to $C_{m-1}$, coincide with $x^{m-1}$. Finally, we have that

$$
\begin{aligned}
& \frac{1}{((m-1) !)^{n} \cdot m^{3 n}} \sum_{x \in L\left(C_{m}\right)^{n}}\left|A\left(\left.x\right|_{C_{m-1}}\right)\right| \cdot\left|B\left(\left.x\right|_{C_{m-1}}\right)\right| \\
= & \frac{1}{(m !)^{n}} \sum_{x \in L\left(C_{m}\right)^{n}} \frac{\left|A\left(\left.x\right|_{C_{m-1}}\right)\right|}{m^{n}} \cdot \frac{\left|B\left(\left.x\right|_{C_{m-1}}\right)\right|}{m^{n}} \\
= & E_{x}\left[\frac{\left|A\left(\left.x\right|_{C_{m-1}}\right)\right|}{m^{n}} \cdot \frac{\left|B\left(\left.x\right|_{C_{m-1}}\right)\right|}{m^{n}}\right]
\end{aligned}
$$

as claimed.

Given any $X \subseteq\{1, \ldots, m\}^{n}$, we define the upper edges of $X$ in direction $i$ to be the set of pairs $\left(v, v_{i}^{\prime}\right)$ such that $v \in X$, and after increasing the $i$ th component of $v$ to $v_{i}^{\prime}$, the resulting element will be out of $X$.

Definition 7 For any $X \subseteq\{1, \ldots, m\}^{n}$, any $i \leq n$, let the upper edges $\partial_{i}(X)$ in direction $i$ be defined as follows:

$$
\begin{aligned}
\partial_{i}(X) & =\left\{\left(v_{-i}, v_{i}, v_{i}^{\prime}\right): v_{-i} \in\{1, \ldots, m\}^{n-1},\right. \\
& \left.v_{i}, v_{i}^{\prime} \in\{1, \ldots, m\},\left(v_{-i}, v_{i}\right) \in X,\left(v_{-i}, v_{i}^{\prime}\right) \notin X, v_{i}<v_{i}^{\prime}\right\}
\end{aligned}
$$

Also, let $\partial(X)=\bigcup_{i=1}^{n} \partial_{i}(X)$.

We now make the following claim to relate $M_{i, m, n}(r)$ to $\partial_{i}\left(A\left(\left.x\right|_{C_{m-1}}\right)\right)$ and $\partial_{i}\left(B\left(\left.x\right|_{C_{m-1}}\right)\right)$. 


\section{Claim 3}

$M_{i, m, n}(r) \geq \frac{1}{m !} m^{-n} E_{x}\left[\left|\partial_{i}\left(A\left(\left.x\right|_{C_{m-1}}\right)\right)\right|+\left|\partial_{i}\left(B\left(\left.x\right|_{C_{m-1}}\right)\right)\right|\right]$

Proof of Claim 3: First, given a vector $x^{m-1}$ of orders of $C_{m-1}$, we define an injective function $g_{i}$ that maps every $\left(v_{-i}, v_{i}, v_{i}^{\prime}\right) \in$ $\partial_{i}\left(A\left(x^{m-1}\right)\right)$ (where $v_{i}, v_{i}^{\prime}$ are positions in $\{1, \ldots, m\}$, with $v_{i}<$ $v_{i}^{\prime}$, and $v_{-i}$ is a vector of $n-1$ such positions) to a successful manipulation $g_{i}^{c_{1}}\left(x^{m-1}, v_{-i}, v_{i}, v_{i}^{\prime}\right)=\left(P, V_{i}^{\prime}\right)$ for voter $i$, where $P$ is a profile representing the agents' true preferences, and $V_{i}^{\prime}$ is the manipulation for voter $i$. Let $r\left(M\left(x^{m-1}, v_{-i}, v_{i}^{\prime}\right)\right)=c_{j}$, where $j \neq 1$. Then, let

$$
\begin{aligned}
& g_{i}^{c_{1}}\left(x^{m-1}, v_{-i}, v_{i}, v_{i}^{\prime}\right) \\
& =\left\{\begin{array}{r}
\left(M\left(x^{m-1}, v_{-i}, v_{i}\right), M\left(x^{m-1}(i), v_{i}^{\prime}\right)\right), \\
\text { if } c_{j} \succ_{M\left(x^{m-1}(i), v_{i}\right)} c_{1} \\
\left(M\left(x^{m-1}, v_{-i}, v_{i}^{\prime}\right), M\left(x^{m-1}(i), v_{i}\right)\right), \\
\text { if } c_{1} \succ_{M\left(x^{m-1}(i), v_{i}\right)} c_{j}
\end{array}\right.
\end{aligned}
$$

That is, we consider the order $M\left(x^{m-1}(i), v_{i}\right)$ that results from taking the $i$ th order in $x^{m-1}$, and inserting $c_{m}$ in position $v_{i}$. If, in this order, $c_{j}$ is ranked higher than $c_{1}$, then we make $M\left(x^{m-1}(i), v_{i}\right)$ the true preference of $i$, and $M\left(x^{m-1}(i), v_{i}^{\prime}\right)$ (where we place $c_{m}$ in the $v_{i}^{\prime}$ th position instead) $i$ 's false report of his preferences. If $c_{j}$ is ranked lower than $c_{1}$, then we make $M\left(x^{m-1}(i), v_{i}^{\prime}\right)$ the true preference, and $M\left(x^{m-1}(i), v_{i}\right)$ the false report of his preferences. We now show that this manipulation $g_{i}^{c_{1}}\left(x^{m-1}, v_{-i}, v_{i}, v_{i}^{\prime}\right)$ is indeed successful. There are 4 different cases that we need to consider.

1. $j \neq m$ and $c_{j} \succ_{x^{m-1}(i)} c_{1}$ : by changing his vote from $M\left(x^{m-1}(i), v_{i}\right)$ to $M\left(x^{m-1}(i), v_{i}^{\prime}\right)$, voter $i$ changes the outcome from $c_{1}$ to $c_{j}$, and he prefers the latter.

2. $j \neq m$ and $c_{1} \succ_{x^{m-1}(i)} c_{j}$ : by changing his vote from $M\left(x^{m-1}(i), v_{i}^{\prime}\right)$ to $M\left(x^{m-1}(i), v_{i}\right)$, voter $i$ changes the outcome from $c_{j}$ to $c_{1}$, and he prefers the latter.

3. $j=m$ and $c_{m} \succ_{M\left(x^{m-1}(i), v_{i}\right)} c_{1}$ : by changing his vote from $M\left(x^{m-1}(i), v_{i}\right)$ to $M\left(x^{m-1}(i), v_{i}^{\prime}\right)$, voter $i$ changes the outcome from $c_{1}$ to $c_{m}$, and he prefers the latter because his true preference is $M\left(x^{m-1}(i), v_{i}\right)$.

4. $j=m$ and $c_{1} \succ_{M\left(x^{m-1}(i), v_{i}\right)} c_{m}$ : by changing his vote from $M\left(x^{m-1}(i), v_{i}^{\prime}\right)$ to $M\left(x^{m-1}(i), v_{i}\right)$, voter $i$ changes the outcome from $c_{m}$ to $c_{1}$, and he prefers the latter, for the following reason. By assumption, even an agent with preferences $M\left(x^{m-1}(i), v_{i}\right)$ prefers $c_{1}$ to $c_{m}$; and $i$ 's true preferences $M\left(x^{m-1}(i), v_{i}^{\prime}\right)$ are different from $M\left(x^{m-1}(i), v_{i}\right)$ only in that $c_{m}$ is ranked even lower in $M\left(x^{m-1}(i), v_{i}^{\prime}\right)$.

Now, for every $\left(v_{-i}, v_{i}, v_{i}^{\prime}\right) \in \partial_{i}\left(B\left(x^{m-1}\right)\right)$, we can analogously define $g_{i}^{c_{2}}\left(x^{m-1}, v_{-i}, v_{i}, v_{i}^{\prime}\right)$, which is a successful manipulation for voter $i$. Additionally, for any $x^{m-1}, y^{m-1} \in L\left(C_{m-1}\right)^{n}$ such that $x^{m-1} \neq y^{m-1}$, any $\left(v_{-i}, v_{i}, v_{i}^{\prime}\right) \in \partial_{i} A\left(x^{m-1}\right),\left(w_{-i}, w_{i}, w_{i}^{\prime}\right) \in$ $\partial_{i} B\left(y^{m-1}\right)$, we have

$$
g_{i}^{c_{1}}\left(x^{m-1}, v_{-i}, v_{i}, v_{i}^{\prime}\right) \neq g_{i}^{c_{2}}\left(y^{m-1}, w_{-i}, w_{i}, w_{i}^{\prime}\right)
$$

That is, all of the manipulations are distinct. Therefore,

$$
\begin{aligned}
& M_{i, m, n}(r) \\
\geq & \frac{1}{(m !)^{n+1}} \sum_{x^{m-1}}\left(\left|\partial_{i} A\left(x^{m-1}\right)\right|+\left|\partial_{i} B\left(x^{m-1}\right)\right|\right) \\
= & \frac{1}{(m !)^{n+1} \cdot m^{n}} \sum_{x}\left(\left|\partial_{i} A\left(\left.x\right|_{C_{m-1}}\right)\right|+\left|\partial_{i} B\left(\left.x\right|_{C_{m-1}}\right)\right|\right) \\
= & \frac{1}{(m !) \cdot m^{n}} E_{x}\left(\left|\partial_{i} A\left(\left.x\right|_{C_{m-1}}\right)\right|+\left|\partial_{i} B\left(\left.x\right|_{C_{m-1}}\right)\right|\right)
\end{aligned}
$$

(End of the proof of Claim 3.)

Summing over $i$, we obtain

$$
\begin{aligned}
& \sum_{i=1}^{n} M_{i, m, n}(r) \\
\geq & \sum_{i=1}^{n} \frac{1}{m !} m^{-n} E_{x}\left[\left|\partial_{i}\left(A\left(\left.x\right|_{C_{m-1}}\right)\right)\right|+\left|\partial_{i}\left(B\left(\left.x\right|_{C_{m-1}}\right)\right)\right|\right] \\
= & \frac{1}{m !} m^{-n} E_{x}\left[\left|\partial\left(A\left(\left.x\right|_{C_{m-1}}\right)\right)\right|+\left|\partial\left(B\left(\left.x\right|_{C_{m-1}}\right)\right)\right|\right]
\end{aligned}
$$

Next, we prove an extension of Lemma 8 in [9].

Claim 4 For any disjoint $A, B \subseteq\{1, \ldots, m\}^{n}$, we have $|\partial(A)|+$ $|\partial(B)| \geq m^{-n}|A||B|$.

Proof of Claim 4: The proof is an easy generalization of the proof of Lemma 8 in [9]. The only difference is that in our claim the lattice is $\{1, \ldots, m\}^{n}$ instead of $\{0,1,2\}^{n}$, and the coefficient of the FKG [8] inequality is $m^{-n}$.

We now present the proof in full. $\{1, \ldots, m\}^{n}$ can be organized as a distributive lattice in the following way:

1. $\left(a_{1}, \ldots, a_{n}\right) \leq\left(b_{1}, \ldots, b_{n}\right)$ if and only if for all $j \leq n$, $a_{j} \leq b_{j}$

2. $\left(a_{1}, \ldots, a_{n}\right) \wedge\left(b_{1}, \ldots, b_{n}\right)=\left(\min \left(a_{1}, b_{1}\right), \ldots, \min \left(a_{n}, b_{n}\right)\right)$.

For any $A$, we will find a monotonic set $A^{\prime}$-that is, for any $a \in A^{\prime}$ and $a^{\prime} \succ a, a^{\prime}$ is also in $A^{\prime}$-in this lattice such that $\left|A^{\prime}\right|=|A|$, and there exists a one-to-one correspondence $d$ between $A$ and $A^{\prime}$ with the following properties. For any $a \in A$, there is a path $p(a)$ from $a$ to $d(a)$. (In a path over the lattice, any two adjacent elements $\left(a_{1}, a_{2}\right)$ differ only in one component, and $a_{1}<a_{2}$. For example, $(1,1,1),(1,3,1),(1,3,2)$ is a path.) We will show that for our definitions of $d(a)$ and $p(a)$, for any $a \neq a^{\prime}$, any adjacent pair $a_{1}<a_{2}$ on $p(a)$ and any adjacent pair $a_{1}^{\prime}<a_{2}^{\prime}$ on $p\left(a^{\prime}\right)$, either $a_{1} \neq a_{1}^{\prime}$ or $a_{2} \neq a_{2}^{\prime}$. That is, if we choose one pair of adjacent elements from the path of $a$, and choose another pair of adjacent elements from the path of $a^{\prime}$, then the two pairs of elements are not equal. We will similarly define $d(b)$ and $p(b)$ for any $b \in B$, with the same properties.

To do this, we use a variant of the "component-wise shifting up" from [9]. Given $A$, we will define an $n$-step path, starting at $A_{0}=$ $A$. In step $i$, for any $v_{-i}$, let $N_{v_{-i}}$ denote the set of all elements in $\{1, \ldots, m\}$ that extend $v_{-i}$ to an element of $A_{i-1}$, that is, $N_{v_{-i}}=$ $\left\{v_{i}:\left(v_{i}, v_{-i}\right) \in A_{i-1}\right\}$. Let $n_{v_{-i}}=\left|N_{v_{-i}}\right|$. We also define a function $f_{i}$ such that $f_{i}\left(v_{i}, v_{-i}\right)=\left(m-k+1, v_{-i}\right)$, where $v_{i}$ is the $k$ th largest element in $N_{v_{-i}}$. For example, if $N_{v_{-i}}=\{m-1, m-$ $3, m-4\}$, then $f_{i}\left(m-1, v_{-i}\right)=\left(m, v_{-i}\right), f_{i}\left(m-3, v_{-i}\right)=(m-$ $\left.1, v_{-i}\right), f_{i}\left(m-4, v_{-i}\right)=\left(m-2, v_{-i}\right)$. In other words, $f_{i}$ shifts $\left(N_{v_{\mathbb{I}}-i}, v_{-i}\right)$ up such that $f_{i}\left(N_{v_{-i}}, v_{-i}\right)$ is "locally monotonic", which means that it is monotonic in $\left(\{1, \ldots, m\}, v_{-i}\right)$. Then let $A_{i}=\bigcup_{v_{-i}} f_{i}\left(N_{v_{-i}}, v_{-i}\right)$. Finally, we let $A^{\prime}=A_{n}$. The path of $a$ is defined to be its trace in the shifting-up process, and the end 
of the trace (which is in $A^{\prime}$ ) is defined to be $d(a)$. We note that the length of a path can be less than $n$, because it is possible that in some step $i$, the $i$ th component is not shifted up.

We now prove the property (stated more precisely above) that adjacent pairs of elements in different paths are never equal. Suppose, for the sake of contradiction, that it does not hold. Then, there exist $a \neq a^{\prime}$, an adjacent pair $a_{1}<a_{2}$ in $p(a)$, and an adjacent pair $a_{1}^{\prime}<$ $a_{2}^{\prime}$ in $p\left(a^{\prime}\right)$, such that $a_{1}=a_{1}^{\prime}, a_{2}=a_{2}^{\prime}$. Then, since $a_{1}$ and $a_{2}$ are adjacent, they differ in exactly one component-let us say, the $i$ th component. For any $j \leq n$ and $a \in A$, let $p_{j}(a)$ be the $j$ th element along the trace of $a$, that is, $f_{j}\left(f_{j-1}\left(\ldots f_{1}(a) \ldots\right)\right)$. (We note that, unlike the path starting at $a$, the trace starting at $a$ can have duplicates.) We now know that $a_{1}=a_{1}^{\prime}=p_{i-1}(a)=p_{i-1}\left(a^{\prime}\right)$ and $a_{2}=a_{2}^{\prime}=p_{i}(a)=p_{i}\left(a^{\prime}\right)$ (otherwise, the $i$ th component could not have shifted). However, this is impossible, because for any $j \leq n, f_{j}$ is a one-one function, which implies that for any $a \neq a^{\prime}$ and any $j \leq n, p_{j}(a) \neq p_{j}\left(a^{\prime}\right)$. Thus we have derived the desired contradiction, thereby proving the adjacent-pairs-arenever-equal property. We can similarly prove the property for $B$.

We now prove that $A^{\prime}$ is monotonic. We prove the following claim by induction on the step of the shifting-up process. The claim states that in any step $i \leq n$, for any $\vec{v}_{n-i} \in\{1, \ldots, m\}^{\{i+1, \ldots, n\}}$, the restriction of $A_{i}$ to $\vec{v}_{n-i}$ is monotonic. Here, the restriction of $A_{i}$ to $\vec{v}_{n-i}$, denoted by $\left.A\right|_{\vec{v}_{n-i}}$, is defined as follows:

$$
\left.A_{i}\right|_{\vec{v}_{n-i}}=\left\{\vec{v}_{i} \in\{1, \ldots, m\}^{i}:\left(\vec{v}_{i}, \vec{v}_{n-i}\right) \in A_{i}\right\}
$$

Claim 5 For any $i \leq n$ and any $\vec{v}_{n-i} \in\{1, \ldots, m\}^{\{i+1, \ldots, n\}}$, $\left.A_{i}\right|_{\vec{v}_{n-i}}$ is monotonic.

Proof of Claim 5: We prove the claim by induction. When $i=1$, for any $\vec{v}_{n-1} \in\{1, \ldots, m\}^{\{2, \ldots, n\}},\left.A_{1}\right|_{\vec{v}_{n-1}}$ is monotonic because the first component of each element in $A_{0}=A$ has been shifted up. Now suppose the claim is true for $i=k$; we will show it is true for $i=k+1$. To prove this, we only need to check that given any $\vec{v}_{n-k-1} \in\{1, \ldots, m\}^{k+2, \ldots, n}$, any $\left.\vec{a} \in A_{k+1}\right|_{\vec{v}_{n-k-1}}$, and any $\vec{b}>\vec{a}$ where $\vec{b}$ differs from $\vec{a}$ only in one component, $\left.\vec{b} \in A_{k+1}\right|_{\vec{v}_{n-k-1}}$. We will consider two different cases.

We first consider the case where $\vec{b}$ differs from $\vec{a}$ in the $(k+$ 1)th component. In this case the claim follows directly from the definition of $A_{k+1}$.

We next consider the case where $\vec{b}$ differs from $\vec{a}$ in the $l$ th component, for $l<k+1$. For any $o \leq k+1$, let $a_{o}$ be the oth component of $\vec{a}$, and let $\vec{a}_{-o}=\left(a_{1}, \ldots, a_{o-1}, a_{o+1}, \ldots, a_{k+1}\right)$. We define $b_{o}$ and $\vec{b}_{-o}$ similarly. Then $b_{-(k+1)}>a_{-(k+1)}, a_{l} \neq b_{l}$, and for any $o \neq l, a_{o}=b_{o}$. Specifically, $a_{k+1}=b_{k+1}$. By the definition of $A_{k+1}$, for any $a_{k+1} \leq j \leq m,\left(\vec{a}_{-(k+1)}, j\right) \in A_{k+1}$, which means that there exist $a_{k+1}^{1}, \ldots, a_{k+1}^{m-a_{k+1}+1} \in\{1, \ldots, m\}$ such that for any $1 \leq t \leq m-a_{k+1}+1,\left(\vec{a}_{-(k+1)}, a_{k+1}^{t}, \vec{v}_{n-k-1}\right) \in$ $A_{k}$. Therefore, for any $1 \leq t \leq m-a_{k+1}+1, a_{-(k+1)} \in$ $\left.A_{k}\right|_{\left(v_{n-k-1}, a_{k+1}^{t}\right)}$. Now, the induction assumption states that $\left.A_{k}\right|_{\left(v_{n-k-1}, a_{k+1}^{t}\right)}$ is monotonic; therefore, for any $1 \leq t \leq m-$ $a_{k+1}+1,\left.\vec{b}_{-(k+1)} \in A_{k}\right|_{\left(v_{n-k-1}, a_{k+1}^{t}\right)}$, which means that $\left(\vec{b}_{-(k+1)}, a_{k+1}^{t}, \vec{v}_{n-k-1}\right) \in A_{k}$. Hence, there are more than $m-$ $a_{k+1}$ elements in $N_{\left(\vec{b}_{-(k+1)}, \vec{v}_{n-k-1}\right)}$, which means that

$\vec{b}=\left.\left(\vec{b}_{-(k+1)}, a_{k+1}\right) \in A_{k+1}\right|_{\vec{v}_{n-k-1}}$. Hence, the claim holds for $i=k+1$, and we have proven the induction step. Thus, the claim holds for all $i \leq n$.

(End of the proof of Claim 5.)

Similarly, $B^{\prime}$ is monotonic. Now we can apply the FKG inequality [8] to $A^{\prime}$ and $B^{\prime}$. It follows that $\left|A^{\prime} \cap B^{\prime}\right| / m^{n} \geq\left|A^{\prime}\right| / m^{n}$.
$\left|B^{\prime}\right| / m^{n}=|A| / m^{n} \cdot|B| / m^{n}$. Because $A \cap B=\emptyset$, it follows that $A^{\prime} \cap B^{\prime} \subseteq\left(A^{\prime} \backslash A\right) \cup\left(B^{\prime} \backslash B\right)$, and hence

$|\partial(A)|+|\partial(B)| \geq\left|\left(A^{\prime} \backslash A\right) \cup\left(B^{\prime} \backslash B\right)\right| \geq\left|A^{\prime} \cap B^{\prime}\right| \geq m^{-n}|A||B|$

(End of the proof of Claim 4.)

For any $r$ satisfying all the five conditions, we now apply Inequality (2), Claim 4, and Equation (1) to obtain:

$$
\begin{aligned}
& \sum_{i=1}^{n} M_{i, m, n}(r) \\
\geq & \frac{1}{m !} \cdot m^{-n} E_{x}\left[\left|\partial\left(A\left(\left.x\right|_{C_{m-1}}\right)\right)\right|+\left|\partial\left(B\left(\left.x\right|_{C_{m-1}}\right)\right)\right|\right] \quad \text { Ineq. (2) }
\end{aligned}
$$

$\geq \frac{1}{m !} \cdot m^{-2 n} E_{x}\left[\left|\partial\left(A\left(\left.x\right|_{C_{m-1}}\right)\right)\right| \cdot\left|\partial\left(B\left(\left.x\right|_{C_{m-1}}\right)\right)\right|\right] \quad$ Claim 4

$=\frac{1}{m !} E_{x}\left[\frac{\left|\partial\left(A\left(\left.x\right|_{C_{m-1}}\right)\right)\right|}{m^{n}} \cdot \frac{\left|\partial\left(B\left(\left.x\right|_{C_{m-1}}\right)\right)\right|}{m^{n}}\right]$

$=\frac{1}{m !} M_{n}^{m-1}(r)$

(End of the proof of Lemma 3.)

We are ready to prove the main result.

Proof of Theorem 2: By the Gibbard-Satterthwaite theorem and condition 2 and 3, we know that for any number $n$ of voters, there exists a successful manipulation, which means that for any $n$, $\sum_{i=1}^{n} M_{i, m, n}(r) \geq \frac{1}{(m !)^{n+1}}$. From Lemma 2 and Lemma 3 we know that there exists some $n^{\prime} \in \mathbb{N}$ and a constant $\delta$ (that does not depend on $n$ ) such that for any $n>n^{\prime}, \sum_{i=1}^{n} M_{i, m, n}(r)>$ $\delta$. Therefore, for any $n, \sum_{i=1}^{n} M_{i, m, n}(r)>\min \left\{\frac{1}{(m !)^{n^{\prime}+1}}, \delta\right\}$, and the right-hand side of the inequality is a constant that does not depend on $n$.

\section{APPLYING THE RESULT TO SOME COM- MON VOTING RULES}

In this section, we show that for nontrivial positional scoring rules (with 4 or more alternatives), Copeland (with 5 or more alternatives), STV, maximin, and ranked pairs (all with 3 or more alternatives), the conditions in Theorem 2 hold (thereby showing that those rules are frequently manipulable). (We note that [9] already proves a quite general result for the case of neutral rules with exactly 3 alternatives.) For the results in this section, ties can be broken in any way that is consistent with anonymity. A positional scoring rule is nontrivial, if the components of its scoring vector $(v(1), \ldots, v(m))$ are not all equal, that is, $v(1)>v(m)$.

Proposition 1 Any (nontrivial) positional scoring rule, Copeland, STV, maximin, and ranked pairs satisfy anonymity, homogeneity, every alternative can win, and canceling out. However, Bucklin does not satisfy canceling out.

All that remains to show is that Condition 5 holds for (nontrivial) positional scoring rules, Copeland with 5 or more alternatives, STV, maximin, and ranked pairs. We recall Condition 5: There exists a profile $P$ such that: (a) $P$ and $D_{m}(P)$ are both stable, (b) $r(P)=$ $c_{1}$, and (c) $r\left(D_{m}(P)\right)=c_{2}$.

A positional scoring rule is nontrivial if it is defined by a score vector $\left(s_{1}, \ldots, s_{m}\right)$ for which $s_{1}>s_{m}$.

Proposition 2 For any nontrivial positional scoring rule $r_{s}$, when there are 4 or more alternatives, there exists a profile that satisfies Condition 5 in Theorem 2. 
Proof of Proposition 2: We will consider two cases. The first case is that $s_{2}, \ldots, s_{m}$ are not all equal. In other words, $s_{2}>s_{m}$. In this case, let $k>2$ be the smallest number such that $s_{k}$ is different from $s_{k-1}$. That is, $s_{2}=\ldots=s_{k-1}>s_{k}$. Let the profile $P_{s}$ consists of two parts, $P_{1}$ and $P_{2}$. Let $P_{1}=a\left(c_{1} \succ c_{m} \succ c_{3} \succ\right.$ $\ldots c_{k-1} \succ c_{2} \succ$ others $)+b\left(c_{2} \succ c_{3} \succ \ldots \succ c_{k-1} \succ c_{1} \succ\right.$ $c_{m} \succ$ others), where $a$ and $b$ satisfy the following conditions:

1. $a s_{1}+b s_{2}>a s_{k}+b s_{1}$

2. $b>a$

Since 1 is equivalent to $a\left(s_{1}-s_{k}\right)>b\left(s_{1}-s_{2}\right)$ and $s_{1}-s_{k}>$ $s_{1}-s_{2} \geq 0$, there exist $a, b>0$ that satisfy both conditions. Let $M_{c}$ be the permutation that cycles $\left\{c_{3}, \ldots, c_{m}\right\}$, that is,

$$
M_{c}\left(c_{i}\right)=\left\{\begin{array}{cc}
c_{i} & \text { if } i=1,2 \\
c_{i+1} & \text { if } 3 \leq i \leq m-1 \\
c_{3} & \text { if } i=m
\end{array}\right.
$$

Let $V_{1}=c_{1} \succ c_{2} \succ c_{3} \ldots \succ c_{m}, V_{2}=c_{2} \succ c_{1} \succ c_{3} \ldots \succ c_{m}$. Then, let $P_{2}=a m \sum_{i=0}^{m-3}\left(M_{c}^{i}\left(V_{1}\right)+M_{c}^{i}\left(V_{2}\right)\right)$.

We now show that $P_{s}=P_{1}+P_{2}$ satisfies Condition 5 in Theorem 2. We need to check that $r_{s}\left(P_{s}\right)=c_{1}, r_{s}\left(D_{m}\left(P_{s}\right)\right)=c_{2}$, and both $P_{s}$ and $D_{m}\left(P_{s}\right)$ are stable.

It is easy to check that $r_{s}\left(P_{s}\right)=c_{1}$. We now show that $r_{s}\left(D_{m}\left(P_{s}\right)\right)=c_{2}$. The score of $c_{2}$ in $D_{m}\left(P_{s}\right)$ is higher than the score of $c_{1}$ in $D_{m}\left(P_{s}\right)$, because they get the same score in $D_{m}\left(P_{2}\right), c_{2}$ gets $a\left(\frac{m-k+1}{m} \cdot s_{2}+\frac{k-1}{m} \cdot s_{k}\right)+b\left(\frac{m-1}{m} \cdot s_{1}+\frac{1}{m} \cdot s_{2}\right)$ in $D_{m}\left(P_{1}\right)$, and $c_{1}$ gets $b\left(\frac{m-k+1}{m} \cdot s_{2}+\frac{k-1}{m} \cdot s_{k}\right)+a\left(\frac{m-1}{m} \cdot s_{1}+\frac{1}{m} \cdot s_{2}\right)$ in $D_{m}\left(P_{1}\right)$. Hence, because $b>a$ and $s_{1} \geq s_{2}>s_{k}$, we know that the score of $c_{2}$ is higher than that of $c_{1}$ in $D_{m}\left(P_{s}\right)$.

Also, the score of $c_{2}$ is higher than the score of $c_{m}$, because the score of $c_{2}$ is:

$$
\begin{aligned}
& a\left(\frac{m-k}{m} \cdot s_{2}+\frac{k}{m} \cdot s_{k}\right)+b\left(\frac{m-1}{m} \cdot s_{1}+\frac{1}{m} \cdot s_{2}\right) \\
& +\frac{a m(m-2)}{m}\left((m-1) s_{1}+(m-1) s_{2}+2 s_{3}\right) \\
> & a s_{k}+b\left(\frac{m-1}{m} \cdot s_{1}+\frac{1}{m} \cdot s_{2}\right)+a(m-2)\left((m-1) s_{1}\right. \\
& \left.+(m-1) s_{2}+2 s_{3}\right) \\
= & a\left((m-2)(m-1) s_{1}+(m-2)(m-1) s_{2}+2(m-2) s_{3}+s_{k}\right) \\
& +b\left(\frac{m-1}{m} \cdot s_{1}+\frac{1}{m} \cdot s_{2}\right) \\
\geq & a\left(\left(2 m-4+\frac{1}{m}\right) \sum_{i=1}^{m} s_{i}\right)+b\left(\frac{1}{m} \sum_{i=1}^{m} s_{i}\right) \\
= & \text { the score of } c_{m}
\end{aligned}
$$

The last inequality follows from the facts that $m \geq 4$ and $s_{1} \geq$ $s_{2} \geq \ldots \geq s_{m}$.

Similarly, we can prove that for any $i \geq 3$, the score of $c_{2}$ is higher than the score of $c_{i}$. Since the inequality is strict in each case, we know that $P_{s}$ and $D_{m}\left(P_{s}\right)$ are stable. This completes the proof of the first case $\left(s_{2}>s_{m}\right)$.

In the second case, $s_{1}>s_{2}=\ldots=s_{m}$ (the plurality rule). In this case let $P_{s}=3\left(c_{1} \succ c_{2} \succ c_{m} \succ\right.$ others $)+2\left(c_{2} \succ\right.$ $c_{1} \succ c_{m} \succ$ others $)+2\left(c_{m} \succ c_{2} \succ c_{1} \succ\right.$ others $)$. It is easy to check that $r_{s}\left(P_{s}\right)=c_{1}$ and $r_{s}\left(D_{m}\left(P_{s}\right)\right)=c_{2}$, and for both $P_{2}$ and $D_{m}\left(P_{s}\right)$, the scores of the winners are strictly larger than the scores of other alternatives, which means that $P_{s}$ and $D_{m}\left(P_{s}\right)$ are stable.

So any nontrivial positional scoring rule satisfies Condition 5 in Theorem 2.
Proposition 3 For the Copeland rule with 5 or more alternatives, there exists a profile that satisfies Condition 5 in Theorem 2.

Proof of Proposition 3: The profile is $P_{s}=c_{1} \succ c_{m} \succ c_{2} \succ$ $c_{3} \succ c_{4} \succ$ others $+m\left(c_{2} \succ c_{4} \succ c_{1} \succ c_{m} \succ c_{3} \succ\right.$ others $)+$ $m\left(c_{3} \succ c_{4} \succ\right.$ others $\left.\succ c_{1} \succ c_{m} \succ c_{2}\right)$. It follows that Copeland $\left(P_{s}\right)=c_{1}$. In $D_{m}\left(P_{s}\right)$, the Copeland score of $c_{1}$ is $m-3$ because it only loses to $c_{m}$ and $c_{4}$ in pairwise elections. However, the Copeland score of $c_{2}$ is $m-2$ because it only loses to $c_{1}$. The Copeland score of any other $c_{j}$ is less than $m-2$. So Copeland $\left(D_{m}\left(P_{s}\right)\right)=c_{2}$. Since none of the pairwise elections result in a tie, the result of each pairwise election is not sensitive to a small fraction of additional votes, which means that both $P_{s}$ and $D_{m}\left(P_{s}\right)$ are stable.

Proposition 4 For the maximin, ranked pairs, and STV rules, with 3 or more alternatives, there exists a profile that satisfies condition 5. in Theorem 2.

Proof of Proposition 4: Let $P_{s}=3\left(c_{1} \succ c_{m} \succ c_{2} \succ\right.$ others $)+$ $2\left(c_{m} \succ c_{2} \succ c_{1} \succ\right.$ others $)+2\left(c_{2} \succ c_{1} \succ c_{m} \succ\right.$ others $)$. Then, $\operatorname{STV}\left(P_{s}\right)=\operatorname{Maximin}\left(P_{s}\right)=\operatorname{RankedPair}\left(P_{s}\right)=c_{1}$. In $D_{m}\left(P_{s}\right)$, the minimum pairwise score of $c_{1}$ is no more than 3 , because $c_{1}$ is ahead of $c_{2}$ in only 3 votes. The minimum pairwise score of $c_{2}$ is more than $\frac{11}{3}$ because $c_{2}$ is ahead of $c_{1}$ in 4 votes, and ahead of $c_{m}$ in $3 \cdot \frac{m-2}{m}+2 \cdot \frac{m-1}{m}+2 \cdot \frac{m-1}{m} \geq 3 \cdot \frac{1}{3}+2 \cdot \frac{2}{3}+2 \cdot \frac{2}{3}=\frac{11}{3}$ votes. $c_{2}$ also defeats all the other alternatives $\left(c_{3}, \ldots, c_{m-1}\right)$ in their pairwise elections. So, $c_{2}$ is the Condorcet winner, which means that $\operatorname{Maximin}\left(D_{m}\left(P_{s}\right)\right)=\operatorname{RankedPairs}\left(D_{m}\left(P_{s}\right)\right)=$ $c_{2}$. For STV, in the first round, all of $c_{3}, \ldots, c_{m-1}$ are eliminated; then, in the second round, since $c_{2}$ is ranked first (among the remaining alternatives) the greatest number of times, either $c_{1}$ or $c_{m}$ will be eliminated. In either case $c_{2}$ is still ranked first (among the remaining alternatives) the greatest number of times in the third round. Therefore, $\operatorname{STV}\left(D_{m}\left(P_{s}\right)\right)=c_{2}$. Both $P_{s}$ and $D_{m}\left(P_{s}\right)$ are stable because there is no tie in any pairwise election or in any step of STV.

We summarize the results of this section as follows:

Theorem 3 Any nontrivial positional scoring rule (with 4 or more alternatives), Copeland (with 5 or more alternatives), STV, maximin, and ranked pairs (all of the three rules with 3 or more alternatives) are frequently manipulable.

\section{CONCLUSIONS}

By the Gibbard-Satterthwaite theorem, for any voting rule that satisfies non-dictatorship and non-imposition, if there are three or more alternatives, then there exists a successful manipulation. Recently, a quantitative version of this result was proved by Friedgut et al. [9]. This new theorem relates the distance between a neutral voting rule and the set of dictatorships to the probability that a random manipulation (from a random profile) will succeed, when the number of alternatives is three. (Here, "random" means uniformly random.) Unfortunately, it does not seem that the proof for that result can be easily extended to more than three alternatives.

In this paper, we considered a different (incomparable) set of conditions under which a similar result holds for more than three alternatives. We showed that if a voting rule satisfies homogeneity, anonymity, non-imposition, a canceling-out condition, and a stability condition, then there exists at least one voter such that a random manipulation for this voter will succeed with a probability of $\Omega\left(\frac{1}{n}\right)$. (We note that we did not assume neutrality.) We showed that any positional scoring rule, STV, Copeland with five or more alternatives, maximin, and ranked pairs satisfy these conditions. 
Apart from the fact that it is restricted to three alternatives, the theorem by Friedgut et al. is a very general result about voting rules: it only requires that the voting rule is neutral (and the result depends on the distance to dictatorial rules). The conditions for our theorem are technically incomparable because we do not assume neutrality, but in practice they seem more restrictive: although some common voting rules satisfy them, other rules do not. Therefore, we still consider it an important open problem to generalize the theorem by Friedgut et al. to four or more alternatives, perhaps with some additional assumptions that are less restrictive than the ones in this paper. Additionally, in this paper, we treated $m$, the number of alternatives, as a constant, and because of that we did not consider the dependence of the probability of success on $m$. Another interesting question is how these results generalize to coalitional manipulation. Once all these questions are settled, we should re-evaluate the agenda of preventing manipulation by making it computationally hard.

\section{Acknowledgements}

We thank Ariel Procaccia, Jeff Rosenschein, Noam Nisan, and the anonymous reviewers for helpful feedback on this paper. This work is supported in part by the United States-Israel Binational Science Foundation under grant 2006216. Lirong Xia is supported by a James B. Duke Fellowship and Vincent Conitzer is supported by an Alfred P. Sloan Research Fellowship.

\section{REFERENCES}

[1] John Bartholdi, III and James Orlin. Single transferable vote resists strategic voting. Social Choice and Welfare, 8(4):341-354, 1991.

[2] John Bartholdi, III, Craig Tovey, and Michael Trick. The computational difficulty of manipulating an election. Social Choice and Welfare, 6(3):227-241, 1989.

[3] Vincent Conitzer and Tuomas Sandholm. Universal voting protocol tweaks to make manipulation hard. In Proceedings of the Eighteenth International Joint Conference on Artificial Intelligence (IJCAI), pages 781-788, Acapulco, Mexico, 2003.
[4] Vincent Conitzer and Tuomas Sandholm. Nonexistence of voting rules that are usually hard to manipulate. In Proceedings of the National Conference on Artificial Intelligence (AAAI), Boston, MA, 2006.

[5] Vincent Conitzer, Tuomas Sandholm, and Jérôme Lang. When are elections with few candidates hard to manipulate? Journal of the ACM, 54(3):Article 14, 1-33, 2007.

[6] R. M. Dudley. Real Analysis and Probability, 2nd Edition. Cambridge University Press, 2002.

[7] Edith Elkind and Helger Lipmaa. Hybrid voting protocols and hardness of manipulation. In Annual International Symposium on Algorithms and Computation (ISAAC), 2005.

[8] C. M. Fortuin, P. W. Kasteleyn, and J. Ginibre. Correlation inequalities on some partially ordered sets. Communications in Mathematical Physics, 22(2):89-103, June 1971.

[9] Ehud Friedgut, Gil Kalai, and Noam Nisan. Elections can be manipulated often, 2007.

[10] Allan Gibbard. Manipulation of voting schemes: a general result. Econometrica, 41:587-602, 1973.

[11] Edith Hemaspaandra and Lane A. Hemaspaandra. Dichotomy for voting systems. Journal of Computer and System Sciences, 73(1):73-83, 2007.

[12] Ariel D. Procaccia and Jeffrey S. Rosenschein. Junta distributions and the average-case complexity of manipulating elections. Journal of Artificial Intelligence Research (JAIR), 28:157-181, 2007.

[13] Mark Satterthwaite. Strategy-proofness and Arrow's conditions: Existence and correspondence theorems for voting procedures and social welfare functions. Journal of Economic Theory, 10:187-217, 1975.

[14] Michael Zuckerman, Ariel D. Procaccia, and Jeffrey S. Rosenschein. Algorithms for the coalitional manipulation problem. In Proceedings of the Annual ACM-SIAM Symposium on Discrete Algorithms (SODA), 2008. 